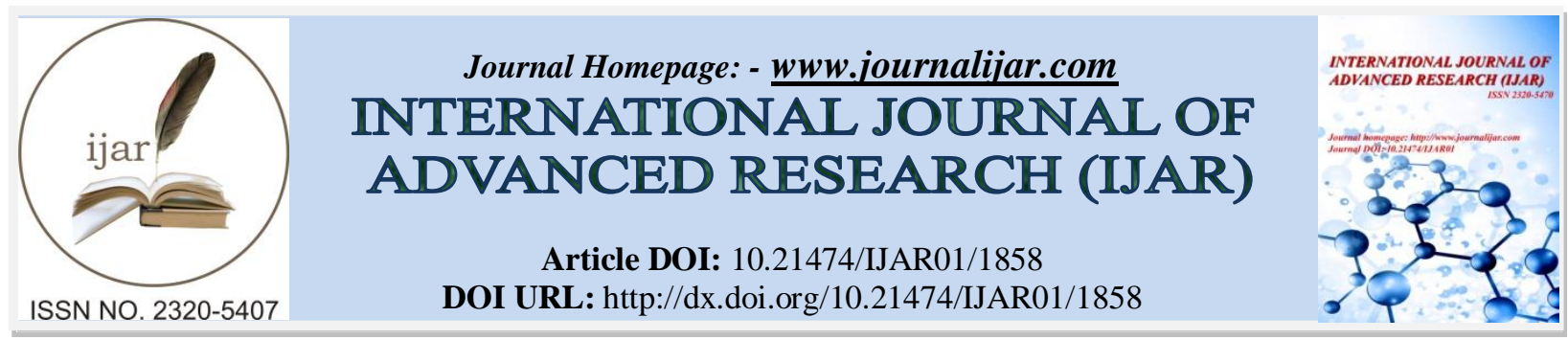

RESEARCH ARTICLE

\title{
TUMEUR DESMOIDE DE LOCALISATION INTRA-THORACIQUE : A PROPOS D'UN CAS. DESMOID TUMOR INTRATHORACIC LOCATION: ABOUT A CASE.
}

N. Hammoune, H. Doulhousne, R. Roukhsi, A. Mouhsine, M. Mahfoudi and A. Elfikri.

Service de radiologie, Hopital militaire Avicenne, Marrakech, Maroc.

\section{Manuscript Info}

Manuscript History

Received: 12 August 2016

Final Accepted: 22 September 2016

Published: October 2016

Key words:-

Desmoid tumor - fibromatosis -

intrathoracic.

\section{Abstract}

Desmoid tumors or fibromatosis are rare and benign fibroblast soft tissue tumors, characterized by infiltrating invasive power, a very high rate of local recurrence but without metastatic potential. Their intrathoracic localization is extremely rare. We describe a case of patient with a desmoid tumor intrathoracic multifocal with a location at the root of the right arm.

Copy Right, IJAR, 2016,. All rights reserved.

\section{Introduction:-}

Les tumeurs desmoïdes sont des tumeurs fibroblastiques agressives bénignes et rares des tissus mous. Elles siègent habituellement au niveau abdominal, la racine des membres et la paroi thoracique. La localisation médiastinale est extrêmement rare. Le diagnostic de certitude est histologique. L'imagerie, peu évocatrice du diagnostic, permet de faire le bilan lésionnel et surtout de guetter les récidives. Le traitement repose idéalement sur la chirurgie lorsqu'elle est possible.

\section{Observation:-}

Il s'agissait d'un patient âgé de 48 ans, ayant comme antécédent un traumatisme thoracique il ya 20 ans avec notion de contusions pulmonaires et des parties molles dorsales droites, qui accusait depuis 5 ans des douleurs scapulaires droites gênant la mobilisation de l'épaule droite évoluant dans un contexte d'apyrexie et de conservation de l'état général. L'examen clinique avait objectivé une voussure du bord axillaire de l'omoplate droite sans signes inflammatoires en regard. La radiographie standard montrait une opacité para-cardiaque droite grossièrement arrondie et une lésion ostéolytique du bord axillaire de l'omoplate droite (figure 1). Le complément tomodensitométrique révélait la présence d'un processus médiastino-pulmonaire droit, de nodules pulmonaires et d'un processus ostéolytique de l'épine de l'omoplate droite envahissant par contiguïté les muscles avoisinants sus et sous épineux (figures 2 et 3). La fibroscopie bronchique ne révélait pas de signes de malignité. L'étude anatomopathologique d'une biopsie chirurgicale réalisée au niveau de la masse sous scapulaire, qui était à l'ouverture charnue et blanchâtre, était en faveur d'une tumeur fibromyoblastique confirmée par l'immuno-histochimie. Des biopsies de la masse médiastinale inferieure ainsi qu'une résection en coin emportant un nodule parenchymateux ont été réalisés par mini thoracotomie. L'étude histologique et l'immuno-histochimique étaient aussi en faveur d'une tumeur desmoïde alors que le nodule parenchymateux s'est avéré être un neurofibrome bénin. Devant la non resécabilité de la tumeur médiastinale, l'importance des champs à irradier contre-indiquant la radiothérapie et la conservation de l'état général, une simple surveillance avec traitement symptomatique ont été optés. L'IRM thoracique réalisée après une année a objectivé la stabilité des différentes lésions citées (figures 4 et 5). 


\section{Discussion:-}

Les tumeurs desmoïdes ou fibromatoses agressives sont des tumeurs bénignes et rares, développées à partir du tissu conjonctif de l'appareil musculo-aponévrotique (1). Elles représentent 3,5\% des tumeurs des tissus mous et 0,03\% de l'ensemble des tumeurs (2). Elles siègent habituellement au niveau abdominal. Les formes extra-abdominales se voient aux ceintures, à la racine des membres, plus rarement au thorax. Leur étiologie demeure incertaine. La prédilection chez la femme jeune entre 25 et 35 ans, la régression spontanée après ménopause ou traitement ostrogénique suggère la cause hormonale. D'autres facteurs favorisants sont incriminés, notamment, les antécédents de chirurgie, de traumatismes ou certains facteurs génétiques (3). La localisation médiastinale primitive est extrêmement rare, elle survient le plus souvent par extension intra-thoracique d'une tumeur pariétale ou cervicale (4). Le tableau clinique est peu spécifique et dépend de la localisation et du pouvoir infiltrant de la tumeur pouvant atteindre les gros vaisseaux, la plèvre, les cotes, les nerfs et le péricarde. Il peut s'agir de douleurs thoraciques et/ ou de signes de compression des structures de voisinage (syndrome de veine cave, insuffisance cardiaque....). L'imagerie est non spécifique et ne permet pas le diagnostic. La TDM met en évidence une masse plus au moins bien limitée de densité tissulaire, iso ou discrètement hypodense, se rehaussant de façon homogène, parfois hétérogène dans les tumeurs volumineuses avec des plages de nécrose et microhémorragies. L'IRM permet une meilleure caractérisation tissulaire, une appréciation meilleure des rapports avec les structures avoisinantes (nerfs, vaisseaux, organes profonds) et la différenciation des remaniements postopératoires ou après traitement médical d'une récidive tumorale (6). Il s'agit souvent d'une masse ovoïde ou infiltrante, à limites généralement lobulées ou parfois irrégulières, présentant un iso ou hyposignal homogène en séquence pondérée $\mathrm{T} 1$ et un signal variable souvent en hypersignal en séquence $\mathrm{T} 2$, la prise de contraste est intense et hétérogène après injection de gadolinium. La présence de bandes en hyposignal, en rapport avec des faisceaux de collagène, sur toutes les séquences est très caractéristique (6). Le diagnostic différentiel se fait selon la localisation avec les autres tumeurs médiastinales surtout les sarcomes et les lymphomes. Le diagnostic de certitude est anatomopathologique par la mise en évidence de cellules fibroblastiques ou myofibroblastiques confirmé par l'étude immuno-histochimique. Le traitement est essentiellement chirurgical avec une résection large lorsqu'elle est possible. En cas de non resécabilité ou de contreindication, le traitement médical fait appel à l'hormonothérapie et aux anti-inflammatoires pour stabilisation voire plus rarement une régression de la taille de la tumeur. Des cures de chimiothérapie ou encore une radiothérapie externe peuvent être proposées dans les formes agressives, résistantes au traitement médical ou inextirpables (7-8).

\section{Conclusion:-}

La localisation médiastinale des tumeurs desmoïdes est une entité très rare. Son diagnostic doit être évoqué devant toute masse intra-thoracique avec antécédent de traumatisme ou de chirurgie thoracique. Le diagnostic de certitude est histologique. L'imagerie est peu spécifique. Le pronostic est imprévisible, il dépend de la localisation et du caractère infiltrant de la tumeur.

\section{Conflit d'interet:-}

Les auteurs déclarent ne pas avoir de conflit d'intérêt avec cet article.

\section{Les figures:-}

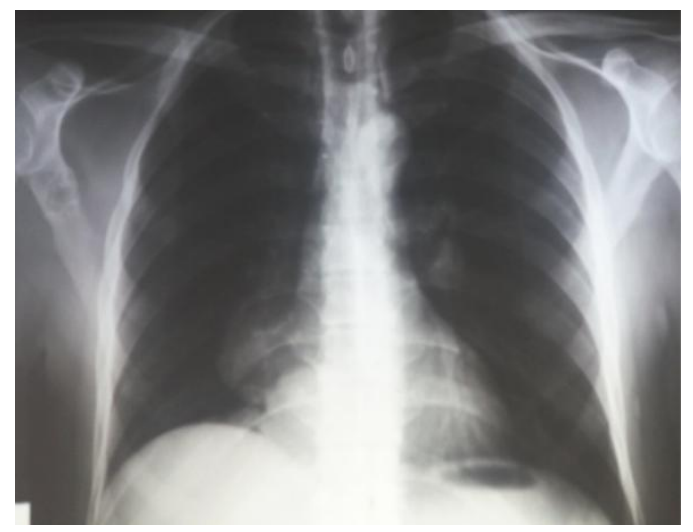

Figure n 1:- radiographie du thorax de face montrant une opacité para cardiaque droite et une lyse du bord axillaire de l'omoplate droite. 


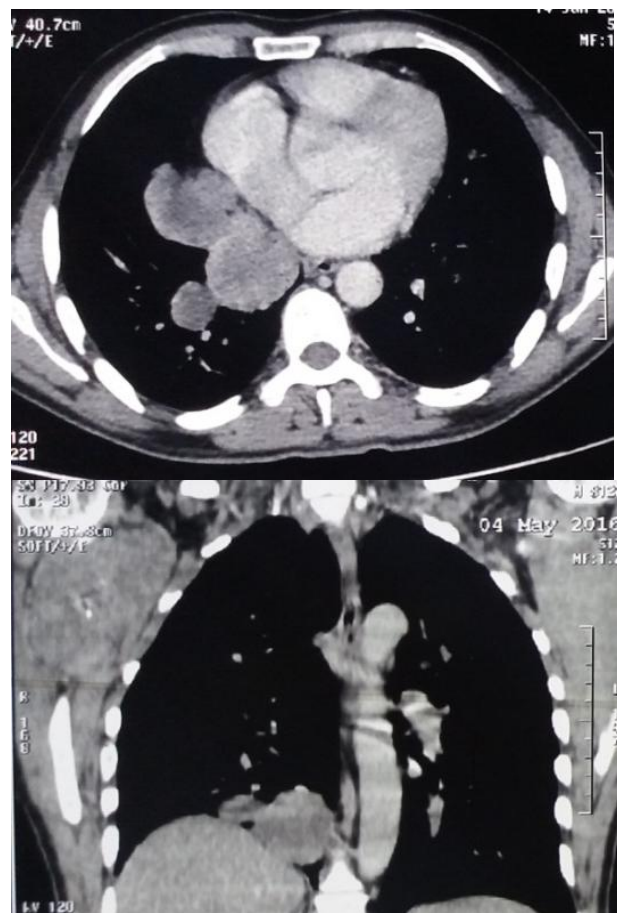

Figure $\mathbf{n} 2$ et 3:- Aspect TDM (coupes axiale et coronale) de trois formations tissulaires hypodenses para cardiaques droites avec processus lytique de l'épine de l'omoplate droite.

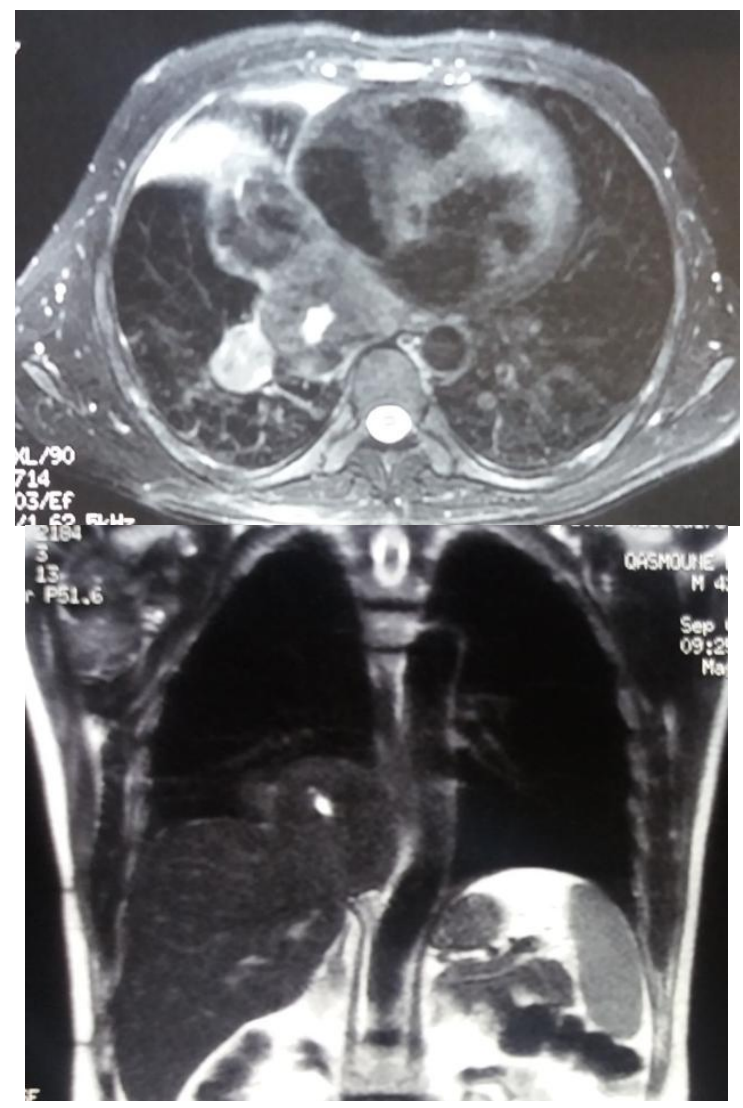

Figures 4 et 5:- masses médiastinales droites de signal hétérogène avec processus lytique de l'omoplate droite en séquences T2 FSE (coupe axiale) et (coupe coronale). 


\section{Les References:-}

1. Shimosato Y, Mukai K : Tumors of mediastinum; in Atlas of Tumor Pathology. Washington, Armed Forces Institute of Pathology 1997;21:249-52.

2. Goldblum J, Fletcher JA : Desmoid-type fibromatoses. World Health Organization: Classification of Tumors. Pathology and Genetics of Tumors of soft Tissue and Bone. Lyon, IARC Press 2002 : 83-4.

3. Enzinger FM, Weiss SW : Fibromatosis. In : Enzinger FM, Weiss SW, eds. Soft Tissue Tumors. $4^{\text {th }}$ Edition, St, Louis, MO, Mosby 2001; 320-9.

4. Harry P, Reitamo JJ, Totterman S, Hopfer-Hallikainen D, Siluva A : The desmoid tumors. II: Analysis of factors possibly contributing to the etiology growth behavior. Ann J Clin Pathol 1982; 77: 674-80.

5. Black WC, Armstrong P, Daniel TM, Cooper PH : Computed tomography of aggressive fibromatosis in the posterior mediastinum. J Comput Assist Tomogr 1987; 11: 153-5.

6. Cotte E, Glehen O, Monneuse O, Cotton F, Vignal J. [Desmoid tumors in familial adenomatous polyposis]. Gastroenterol Clin Biol. 2004;28:57481.

7. Sakorafas G, Nissotakis C, Peros G. Abdominal desmoid tumors. Surg Oncol. 2007;16:13142.

8. Stoeckle E, Coindre J, Longy M, Binh M, Kantor G, Kind M, et al. A critical analysis of treatment strategies in desmoid tumours: a review of a series of 106 cases. Eur J Surg Oncol. 2009;35:12934 